\title{
CONHECIMENTO DE ADOLESCENTES MULHERES SOBRE CONTRACEPÇÃO
}

\author{
Valéria Silvana Faganello Madureira ${ }^{1}$, Ana Isabel Weber ${ }^{2}$
}

RESUMO: Estudo quantitativo investigou o conhecimento de adolescentes sobre contracepção e foi realizado no segundo semestre de 2006. Os dados foram coletados com um questionário aplicado a 76 meninas com idade entre 13 e 18 anos que manifestaram interesse e obtiveram o consentimento escrito dos pais e/ou responsáveis. As principais fontes de informações sobre contracepção foram os pais, revistas, livros, jornais e profissionais da saúde; a camisinha masculina e a pílula foram os métodos conhecidos pela maioria das participantes. Ilustrando as desigualdades de poder nas relações de gênero, a confiança no parceiro ou a solicitação feita por esse são argumentos aceitos pelas adolescentes para negligenciar o uso do método anticoncepcional. Os resultados confirmaram que conhecer os métodos contraceptivos não é determinante para o seu uso, configurando a necessidade de maiores investimentos na educação sexual das adolescentes.

PALAVRAS-CHAVE: Adolescente; Anticoncepção; Educação em saúde; Gênero e saúde.

\section{THE KNOWLEDGE OF ADOLESCENT WOMEN ON CONTRACEPTION}

ABSTRACT: This quantitative study investigated adolescents' knowledge of contraception and was carried out in the second semester of 2006.The data were collected from a questionnaire given to 76 girls aged between 13 and 18 who had shown interest and who had been given written permission by parents or guardians. The main sources of information about contraception were the parents, magazines, books, newspapers and health professionals; the male condom and the Pill were the methods known to the majority of the participants. Illustrating gender power inequalities, confidence in the male partner or, simply, a request made by him are arguments accepted by female adolescents for neglecting the use of methods of contraception. The results confirm that simply knowing about methods of contraception is not determinant to their use, indicating that it is appropriate to make greater investments in adolescents' sexual education.

KEYWORDS: Adolescent; Contraception; Health education; Gender and health.

\section{CONOCIMIENTO DE ADOLESCENTES MUJERES SOBRE ANTICONCEPCIÓN}

RESUMEN: Estudio cuantitativo que ha investigado el conocimiento de adolescentes sobre anticoncepción y fue realizado en el segundo semestre de 2006. Los datos fueron recogidos con un cuestionario aplicado a 76 chicas con edad entre 13 y 18 años que manifestaron interés y tuvieron el consentimiento escrito de los padres y/o responsables. Las principales fuentes de información sobre contracepción fueron los padres, revistas, libros, periódicos y profesionales de la salud; el preservativo masculino y a píldora fueron los métodos conocidos por la mayoría de las participantes. Ilustrando las desigualdades de poder en las relaciones de género, la confianza en el compañero o la solicitación hecha por él son argumentos aceptos por las adolescentes para no usar método anticoncepcional. Los resultados confirmaron que conocer los métodos anticonceptivos no es determinante para su uso, configurando la necesidad de más inversiones en la educación sexual de las adolescentes.

PALABRAS CLAVE: Adolescente; Anticoncepción; Educacion en salud; Género y salud.

\footnotetext{
${ }^{1}$ Enfermeira. Doutora em Enfermagem. Professora da Universidade do Contestado - UnC. Líder do Grupo de Estudos e Pesquisas em Promoção da Saúde - GEPEPS.

${ }^{2}$ Enfermeira. Especialista em Obstetrícia. Supervisora de Enfermagem da Fundação Universidade do Oeste de Santa Catarina.
}

\author{
Autor correspondente: \\ Valéria Silvana Faganello Madureira \\ Universidade do Contestado \\ R. Rua Liberal Brezolla, 30 - 89700-000 - Concórdia-SC-Brasil \\ E-mail: valeriamadureira2005@hotmail.com
}

Recebido: $17 / 12 / 10$

Aprovado: $29 / 04 / 11$ 


\section{INTRODUÇÃO}

A adolescência é um período de autoafirmação diante dos adultos no qual alterações físicas, psicológicas e sociais demonstram que meninos e meninas já não são crianças. Nesta transição para a vida adulta ocorrem muitas transformações físicas e psicossociais ${ }^{(1-2)}$. As especificidades emocionais e comportamentais se refletem na saúde sexual e reprodutiva tornando "os jovens mais vulneráveis aos mesmos riscos a que muitos adultos estão expostos"(3:136). Nesta fase, a sexualidade desperta conferindo importância à contracepção, especialmente considerando-se as estatísticas brasileiras de gravidez e de doenças sexualmente transmissíveis (DST/Aids) na adolescência.

Diferentes estudos mostram que nenhuma geração de adolescentes foi tão bem informada sobre sexo quanto a atual ${ }^{(4-6)}$. No entanto, indistintamente de classe social e apesar das informações, os jovens não sabem como usá-las ou aplicá-las na prática. Com a possibilidade de iniciar as atividades sexuais, é preciso escolher um método anticoncepcional, o que geralmente não ocorre, e os jovens iniciam a vida sexual sem se proteger ${ }^{(7-8)}$. Como reflexo, a gravidez entre adolescentes cresceu no Brasil, contrariando a tendência geral de diminuição das taxas de fecundidade no país ${ }^{(9)}$.

Fatores como o conhecimento sobre os métodos disponíveis, sobre os riscos de uma gravidez, a vivência sexual e o relacionamento afetivo-sexual estão envolvidos na escolha de um método contraceptivo (MAC), o que torna a escolha "ainda mais complexa para o adolescente"(4:294).

Os vários tabus que cercam a contraconcepção e a sexualidade na adolescência geram sentimentos de culpa e vergonha nas adolescentes sexualmente ativas, fazendo com que muitas não busquem informações e métodos para prevenir gravidez e DST/Aids. Sexualidade e comportamento sexual estão cercados por valores, tabus e preconceitos fomentados por um meio que espera das mulheres uma conduta sexual contida, enquanto do rapaz espera um desempenho sexual ativo e exacerbado. Em outras palavras, esse campo é impregnado pelas questões de gênero.

O sistema de gênero é um conjunto de práticas, símbolos, representações, normas e valores sociais que as sociedades elaboram a partir da diferença sexual e que dão sentido à satisfação dos impulsos sexuais, à reprodução da espécie humana e ao relacionamento entre as pessoas ${ }^{(10)}$. Nesse sistema, a sexualidade tem aspectos fundamentais na construção da identidade pessoal. Parece haver marcos socialmente organizados no aprendizado das relações que levam à sexualidade adulta, estabelecendo regras de comportamento e atribuindo status aos parceiros e, nesse contexto, o tradicional namoro vem sendo substituído ou complementado pelo ficar ${ }^{(11)}$.

No que se refere às parcerias para a iniciação sexual também há contrastes, pois enquanto entre os homens a diversidade de trajetórias e parcerias é comum, entre as mulheres há homogeneidade na experiência com namorados mais velhos e experientes ${ }^{(12)}$. Para os homens, a iniciação sexual é considerada prova de masculinidade heterossexual, podendo ocorrer com qualquer parceira e o mais cedo possível ${ }^{(11)}$. Para as mulheres, a iniciação sexual é tradicionalmente interpretada como parte de um relacionamento ou na construção de um relacionamento estável. Assim, "a principal prova de feminilidade na adolescência é a capacidade de estabelecer uma relação estável com um membro do sexo

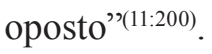

A primeira relação sexual é considerada pelas mulheres como decisiva em um relacionamento verdadeiro, enquanto, para os homens é uma iniciação pessoal na qual o relacionamento com a parceira tem pouca importância ${ }^{(7)}$. Na iniciação sexual, o uso de métodos contraceptivos precisa ser considerado. Entre rapazes, é comum um maior número que faz uso da camisinha, fato possivelmente associado a sexo eventual com parceiras pouco conhecidas ${ }^{(7)}$. Sobre esse aspecto, é necessário informar às meninas sobre os diferentes métodos anticoncepcionais e sobre sua vulnerabilidade em relações sexuais desprotegidas. Assim, esse estudo foi desenvolvido com o objetivo de verificar o conhecimento sobre contracepção entre adolescentes mulheres.

\section{METODOLOGIA}

Este estudo foi desenvolvido em Escola Estadual de Educação Básica, no Estado de Santa Catarina, com o maior número de estudantes do sexo feminino que desejaram participar e que atenderam aos critérios de inclusão: idade entre 13 e 18 anos, e apresentar o Termo de Consentimento Livre e Esclarecido assinado por pais ou responsáveis, o que resultou em 76 sujeitos no estudo.

Os dados foram coletados na escola, nos turnos matutino e vespertino, e durante dois meses de 2006. Um questionário elaborado pelas pesquisadoras, com perguntas abertas e fechadas, foi usado como instru- 
mento de coleta. O mesmo foi previamente testado com três adolescentes e, a partir das respostas obtidas, procedeu-se as modificações necessárias na questão número cinco. Os dados foram organizados, categorizados de acordo com as perguntas do questionário e discutidos com base na literatura. O projeto foi aprovado pelo Comitê de Ética em Pesquisa da Universidade do Contestado - UnC sob protocolo n. 134/2006.

\section{RESULTADOS}

A idade média das participantes foi de 15 anos, a maioria cursava o Ensino Médio $(72,4 \%)$ e a minoria $(13,2 \%)$ estudava na $7^{\mathrm{a}}$ série do Ensino Fundamental. Sobre a renda familiar, 43 adolescentes $(56,5 \%)$ informaram ser de dois a três salários mínimos (SM). Uma renda superior a quatro SM foi informada por $26,3 \%$, enquanto $17,1 \%$ tinham renda mensal de um SM.

A maioria das adolescentes (47) declarou-se solteira e sem namorado no momento. Entre as adolescentes que descreveram sua relação afetiva como "ficando" (9), a maioria tinha idade entre 13 e 16 anos; entre as 29 meninas envolvidas em relacionamento afetivo, 20 afirmaram estar namorando. Das participantes, 26 relataram já terem iniciado sua vida sexual. Sobre a idade na primeira relação, destacou-se a faixa de 14 a 16 anos (24); uma participante revelou ter iniciado sua vida sexual aos 13 anos e outra, aos 17 anos. A idade média de iniciação sexual foi de 14,8 anos.

Entre as adolescentes, $22(84,6 \%)$ escolheram o namorado como parceiro para a iniciação sexual; parceiro não estável e amigos foram citados por quatro adolescentes. Das 26 adolescentes que iniciaram atividade sexual, 25 afirmaram ter usado algum MAC na primeira relação, sendo a camisinha masculina o mais utilizado; 17 continuavam fazendo uso de algum método; 7 relataram não ter parceiro sexual no último semestre e 19 consideraram-se sexualmente ativas, entre as quais 16 relataram monoparceria e três relataram mais de um parceiro sexual no período.

Entre as que já tiveram experiência sexual, 17 afirmaram ter adotado algum MAC em todas as relações, sendo o mais usado a camisinha masculina, indicada por 16 delas. Das 14 adolescentes que prosseguiram usando pílula, $9(64,3 \%)$ relataram uso efetivo da mesma; 8 relataram não-uso de método contraceptivo em todas as relações sexuais e, dentre estas, 7 não tiveram relações no último semestre; seis participantes afirmaram que não teriam coragem de manter relação sexual sem usar MAC; quatro participantes justificaram o não-uso de MAC por não tê-lo consigo no momento ou por esquecimento; a confiança no parceiro foi citada por quatro meninas como motivo para o não-uso de qualquer método.

No que se refere a informações sobre contracepção, os pais foram indicados como primeira fonte; revistas, livros e jornais constituíram a segunda fonte de informação e os profissionais de saúde a terceira. Amigos, televisão, rádio, namorado e irmã constituíram a quarta fonte de informação sobre contracepção. Quanto ao conhecimento sobre MAC, 74 adolescentes declararam conhecer algum, sendo os mais citados, em ordem decrescente, camisinha masculina, pílula, DIU, diafragma e camisinha feminina.

Quanto à confiança no método, 55 adolescentes consideram o preservativo masculino como mais seguro por oferecer dupla proteção e pelas baixas chances de falha; a pílula foi considerada como segundo método mais seguro; cinco participantes consideraram que nenhum método contraceptivo é mais seguro, pois todos podem falhar e quatro consideraram que, quando usados corretamente, todos os métodos são eficazes.

A maioria das adolescentes referiu buscar ou receber informações sobre sexo na família, especialmente com a mãe (52) por lhes dar segurança, ou por maior afinidade porque a mãe se propõe a sanar dúvidas e porque a mãe engravidou na adolescência e não quer que o fato se repita com a filha. A irmã foi apontada como referência por já possuir experiência sexual e pela proximidade de idade. Entre as participantes, 13 relataram que ninguém em sua casa oferece informações sobre sexo. Palestras na escola surgem como maneira mais usada para a disseminação de informações sobre sexo e contracepção, juntamente com as aulas de biologia, colegas e amigas. As participantes revelaram preferência por técnicas como discussão em grupo com pessoas do mesmo sexo, organizadas por outros profissionais e que enfocam abertamente questões relativas a sexo e sexualidade.

Nas questões relativas à contracepção e à iniciação sexual, a maioria $(93,4 \%)$ indicou como verdadeira a opção que afirmava que os preservativos masculino e feminino oferecem proteção para gravidez e DST. As respostas erradas concentraram-se na faixa de 14 a 16 anos. A opção que afirmava a impossibilidade de gravidez na primeira relação sexual, mesmo sem o uso de métodos contraceptivos, foi corretamente indicada como falsa por $90,7 \%$ das adolescentes. A afirmativa de que no período fértil a mulher está ovulando foi indicada como verdadeira por $84,2 \%$ das adolescentes. 
A idéia de que sexo sem penetração não oferece riscos de gravidez e contaminação por DST/Aids foi indicada como falsa por $77,6 \%$ das adolescentes, a maioria com 13 anos. Já $69,7 \%$ reconheceram como verdadeira a possibilidade de gravidez em relação sexual durante o período menstrual. O uso concomitante dos preservativos feminino e masculino para maior segurança foi indicado corretamente como falso por $68,4 \%$ das participantes. Sobre a forma de tomar a pílula anticoncepcional, 68,4\% responderam corretamente à alternativa. A afirmativa que considerava a tabelinha como método eficaz em qualquer idade foi incorretamente identificada como verdadeira por $47,3 \%$ das participantes. A opção que afirmava não ser recomendado guardar a camisinha no bolso ou na carteira para evitar danos foi reconhecida como verdadeira por $53,9 \%$ das participantes.

\section{DISCUSSÃO}

No que se refere ao relacionamento afetivo, a maioria das participantes revelou estar sem namorado no momento da coleta de dados. Sobre isso é importante considerar a possibilidade de muitas manter relacionamentos como o "ficar", referido como um primeiro contato entre o casal e que pode tornar-se namoro. É também uma experiência preliminar, para conhecer melhor o outro. Adolescentes definem "ficar" como "relacionamento episódico e ocasional"(13:71), de caráter breve que, na prática, pode iniciar com carícias, abraços, beijos e evoluir para transa ${ }^{(13)}$, embora não implique compromisso.

A idade média de iniciação sexual (14,8 anos) indica precocidade quando comparada a outros estudos ${ }^{(5,14)}$ e à média brasileira de 15,9 anos para mulheres ${ }^{(15)}$. A esse respeito, é importante considerar as diferenças de gênero na idade de iniciação sexual, pois, enquanto da menina se espera comportamento mais comedido e iniciação sexual tardia, do rapaz se espera ousadia, iniciativa, experiência sexual precoce e variada. Considerando-se as diferenças de gênero, esse dado é relevante, pois há expectativa social de que a iniciação sexual da adolescente seja mais tardia, o que pode ser mais fortemente percebido em cidades do interior, caso da cidade onde a pesquisa foi desenvolvida. Essa norma de gênero se reflete na forma como a sexualidade das mulheres é tratada, especialmente na adolescência, e leva a pensar no silêncio que cerca a iniciação sexual das meninas. Estas, em razão da norma, não têm a quem se referir para buscar esclarecimentos e orien- tações antes da primeira relação sexual sem que se denunciem, expondo-se a recriminações e punições. Essa situação pode aumentar também as possibilidades de envolvimento em uma relação que culmine na iniciação sexual, sem que a adolescente esteja preparada.

A escolha do namorado como parceiro na primeira relação sexual foi encontrada também em outro estudo, no qual 9 entre 10 jovens com vida sexual ativa elegerano como parceiro ideal para a primeira relação sexual referindo que o conheciam melhor e que havia um compromisso amoroso entre ambos ${ }^{(16)}$. Esse resultado está de acordo com as normas de gênero que vinculam as relações sexuais femininas à existência de um vínculo afetivo com o parceiro.

O preservativo masculino foi usado pela maioria das adolescentes na primeira relação sexual; o uso exclusivo da pílula foi feito por três adolescentes. Observa-se um aumento de opção pela pílula e redução no uso do preservativo masculino nas relações subsequentes, o que sugere maior preocupação com prevenção da gravidez do que com sua vulnerabilidade às DST/Aids. Embora o uso da pílula tenha aumentado após a primeira relação sexual, é preocupante o percentual de adolescentes que a utilizam de modo irregular e incorreto e se expõem à DTS/Aids pelo não-uso de preservativo.

Embora muitas jovens afirmaram que não teriam coragem de manter relação sexual sem usar MAC, a confiança no parceiro e a solicitação deste para o não-uso do método, especialmente do preservativo, são situações comumente aceitas pelas adolescentes para negligenciar o uso de contraceptivos. Isso denota a influência do poder masculino sobre as decisões sexuais e reprodutivas da mulher, bem como o tanto que a sexualidade dela depende do outro. A confiança da adolescente no parceiro também foi indicada como justificativa pelo menor número de adolescentes que não utilizava MAC nas suas relações sexuais. É importante ressaltar que a confiança no parceiro não está relacionada ao tempo do relacionamento ou conhecimento mútuo, mas à identificação do relacionamento como namoro.

Sobre a monoparceria sexual nos seis meses anteriores à coleta de dados, referida pela maioria das adolescentes sexualmente ativas, é preciso considerar a possibilidade de que admitir um maior número de parceiros depõe contra a mulher. Múltiplos parceiros pode contribuir para classificá-la como fácil, especialmente em pequenas cidades, onde a vigilância social sobre o comportamento individual é mais intensa. Além disso, 
entre adolescentes com multiparceria sexual há maior exposição e maior vulnerabilidade à DST/Aids ${ }^{(17)}$.

Há, então, a possibilidade de as respostas obtidas não retratarem a realidade vivida pelas adolescentes. Entretanto, é positiva a informação de que a maioria das adolescentes adotou MAC em todas as relações e privilegia o preservativo masculino, pois pode indicar maior consciência da necessidade de adoção de um método eficaz na prevenção de gestação e também de DST/Aids. Quando usado corretamente e em todas as relações sexuais, o preservativo masculino é $97 \%$ eficaz no primeiro ano de uso ${ }^{(18)}$. É possível também que a decisão pelo uso do preservativo em todas as relações seja tomada pelo parceiro. O elevado uso de camisinha masculina encontrado neste e em outros estudos ${ }^{(4-6 ; 14)}$ se deve, provavelmente, às inúmeras campanhas educativas desenvolvidas nos últimos anos pelo Programa Nacional de DST/Aids, com ênfase na prevenção dessas doenças e pelo oferecimento gratuito de preservativos na rede pública de saúde.

Apesar de ser o método de primeira escolha da maioria das adolescentes após a primeira relação sexual, a pílula foi identificada por elas como o segundo método mais seguro. O grau de segurança foi condicionado à utilização correta da mesma, aspecto responsável pela obtenção de bons resultados na prevenção de gravidez. Esse método é muito eficaz quando usado diariamente e de maneira correta, sendo de $0,1 \%$ a possibilidade de falha no primeiro ano de uso $^{(18)}$. As adolescentes reconhecem a função de dupla proteção dos preservativos masculino e feminino, pois os indicam como métodos contraceptivos e de proteção para DST/Aids. A associação entre uso da camisinha e prazer proporcionado pela segurança do seu uso efetivo pode estimular a utilização nas relações sexuais, pois o não-uso equivale a expor-se ao risco de gravidez ou de doença ${ }^{(17)}$.

Os dados indicam também que as participantes atribuíram confiança a métodos como a tabelinha (Ogino-Knaus, método do ritmo) que, mesmo corretamente utilizado, não oferece alta segurança, com índice de falha de $9 \mathrm{em} 100$ mulheres, e com tendência a se elevar em ciclos menstruais irregulares ${ }^{(18)}$. Esse método, baseado no cálculo do período fértil da mulher a partir da data dos últimos ciclos menstruais, lhe permite evitar relações sexuais nesse período ${ }^{(19)}$, mas não é recomendado na adolescência devido à irregularidade dos ciclos menstruais, ao alto índice de falhas e por não oferecer proteção para DST/Aids.

A decisão pelo não-uso de MAC em todas as rela- ções sexuais é tomada após avaliação que a adolescente faz do parceiro e que o indica como confiável ou livre de DST/Aids. Esse julgamento baseia-se na aparência e/ ou no afeto que a adolescente nutre pelo companheiro. Estudo desenvolvido na Bahia identificou que 14,9\% das adolescentes com idade entre 11 e 19 anos justificaram o não-uso de MAC porque desejavam a gravidez e entre 3,8\% das adolescentes porque os parceiros não quiseram usar ${ }^{(14)}$. Em associação com o encontrado no presente estudo, isso remete novamente à posição social de homens e mulheres, a qual se estende às trocas afetivo-sexuais, onde o poder da decisão é do homem.

A busca de orientação sexual com os pais, especialmente com a mãe, é um dado relevante, pois o diálogo na família e a demonstração de afetividade contribuem para diminuir o risco de adoção de comportamentos perigosos ou autodestrutivos ${ }^{(16)}$. A procura dos pais/ mãe para esclarecer dúvidas implica revelar segredos da sexualidade e superação da timidez para fazer perguntas. Na obtenção de informações sobre sexo, revistas, livros e jornais também trazem informações detalhadas, atualizadas, na maioria das vezes, e confiáveis, utilizando linguagem próxima do universo da adolescente.

A escola promove o estudo de temas ligados à sexualidade e à contracepção como conteúdo das aulas de Biologia e Ciências, o que indica que as informações referem-se mais à anatomia e fisiologia do que propriamente à sexualidade, contracepção e prevenção de DST/Aids. Os profissionais da saúde são parceiros da escola na informação das adolescentes, entretanto, essa parceria não substitui o papel do professor na abordagem da sexualidade. Há referências na literatura de que a transmissão de informações científicas relativas ao corpo humano nas escolas é muitas vezes interpretada erroneamente pelas adolescentes ${ }^{(17)}$. É preciso considerar também que a tarefa da escola é difícil, pois é necessário o equilíbrio: por um lado incentivar a sexualidade normal e, pelo outro, simultaneamente, contê-la ${ }^{(20)}$.

Uma gravidez pode resultar da primeira relação sexual e o conhecimento sobre esse aspecto é indicado pelas adolescentes. Entretanto, os quase $10 \%$ que crêem estar protegidas nessa ocasião, mesmo sem usar MAC, indicam a persistência de mitos sobre a iniciação sexual e a falta de conhecimento sobre o assunto e o próprio corpo.

Embora a maioria das adolescentes demonstre algum conhecimento sobre a possibilidade de gravidez na relação sexual sem penetração em período 
fértil, as respostas erradas reforçam a ideia de que muitas desconhecem a fisiologia sexual masculina e feminina. Esse resultado é importante, especialmente quando comparado ao percentual de participantes que consideram a tabelinha como método contraceptivo confiável, o qual depende do ciclo ovulatório, da identificação das alterações provocadas no corpo e da regularidade do ciclo menstrual.

O percentual de respostas erradas para as questões relativas ao uso concomitante de preservativos masculino e feminino revela a crença de que essa prática aumenta a proteção para gravidez e DST/Aids, sem considerar o maior risco de rompimento decorrente do atrito durante a relação sexual. A forma de uso da pílula é também mal compreendida por um número considerável de participantes, o que contribui para explicar o uso incorreto da mesma por várias adolescentes.

Considerando que as mulheres passam a maior parte de sua vida reprodutiva tentando evitar a gravidez, o uso adequado e correto de métodos contraceptivos é necessário para que possam optar pelo momento de engravidar. Dentre os MAC, as participantes demonstraram conhecer, principalmente, o preservativo masculino e a pílula, embora tenham citado também o DIU, o diafragma e o preservativo feminino. Entretanto, a não-citação do coito interrompido está em dissonância com outros estudos ${ }^{(3-5)}$. A contracepção de emergência (pílula do dia seguinte) também é pouco conhecida pelas adolescentes, o que pode dificultar o acesso a esse método, fato agravado pela dependência financeira que a maioria das adolescentes têm de seus pais.

\section{CONCLUSÃO}

Os resultados desse estudo indicam que as adolescentes têm conhecimento sobre MAC, especialmente sobre preservativo masculino e pílula, porém, de forma desorganizada e geralmente o conhecimento não é traduzido nas decisões sexuais e reprodutivas dessas. Isso fica evidente no papel desempenhado pelo parceiro no uso de preservativo na primeira relação sexual e nas subsequentes. O pouco envolvimento da adolescente nessas decisões está vinculado também às normas de gênero que cercam o exercício sexual feminino e às restrições que determinam o tipo da relação.

Nesse particular, a escola, aceita como uma das principais responsáveis pela educação sexual dos adolescentes, não tem assumido efetivamente essa tarefa.
Isso sugere a carência de preparo dos professores para lidar com temáticas relacionadas à sexualidade, bem como para abordá-las a partir de uma perspectiva de gênero, contribuindo para a desmitificação de estereótipos sexuais, especialmente dos femininos.

A iniciação sexual é um momento importante na vida das mulheres e tem acontecido cada vez mais precocemente, na adolescência, quando a mulher não tem a maturidade, o conhecimento e a autoconfiança necessárias para tomar suas próprias decisões sexuais e reprodutivas que lhe possibilitem uma vida sexual saudável e prazerosa. $\mathrm{O}$ conhecimento sobre seu próprio corpo, questões de gênero, possibilidade de gravidez e de exposição às DST/Aids, bem como sobre as possibilidades de preveni-las é importante para respaldar essas decisões.

Família, escola e profissionais da saúde podem ser parceiros e trabalhar consistentemente na informação, orientação e aconselhamento das adolescentes, fomentando a responsabilidade por suas próprias decisões sexuais. Para isso, é preciso reconhecer a heterogeneidade existente entre adolescentes para elaborar estratégias educativas diferenciadas, respeitando características individuais e/ou grupais.

Nesse sentido, a Enfermagem pode atuar no auxílio a professores em temáticas relacionadas com sexualidade, gênero, reprodução e prevenção de DST/Aids, bem como junto às adolescentes, seja no atendimento individual ou em ações coletivas, e no desenvolvimento de pesquisas nesse campo.

\section{REFERÊNCIAS}

1. Leal AC, Wall ML. Percepções da gravidez para adolescentes e perspectivas de vida diante da realidade vivenciada. Cogitare Enferm. 2005;10(3):44-52.

2. Montes MMV, Alvarez GC, Argote LA, Osorio A, Osorio O. La adolescente grávida: un desafio para la família en el afrontamiento de su autoestima. Cogitare Enferm. 2006;11(2):105-12.

3. Vieira LM, Saes SO, Dória AAB, Goldberg TBL. Reflexões sobre a anticoncepção na adolescência no Brasil. Rev Bras Saúde Matern Infant. 2006;6(1):135-40.

4. Guimarães AMDN, Vieira MJ, Palmeira JA. Informações dos adolescentes sobre métodos anticoncepcionais. Rev Latino-Am Enfermagem. 2003;11(3):293-8.

5. Martins LBM, Paiva LC, Osis MJD, Sousa MH, Pinto Neto AM, Tadini V. Conhecimento sobre métodos

Cogitare Enferm. 2011 Abr/Jun; 16(2):333-9 
anticoncepcionais por estudantes adolescentes. Rev Saúde Pública. 2006;40(1):57-64.

6. Belo MAV, Silva JLP. Conhecimento, atitude e prática sobre métodos anticoncepcionais entre adolescentes gestantes. Rev Saúde Pública. 2004;38(4):479-87.

7. Borges ALV, Schor N. Início da vida sexual na adolescência e relações de gênero: um estudo transversal em São Paulo, Brasil, 2002. Cad Saúde Pública. 2005;21(2):499-507.

8. Ferreira MLSM, Galvão MTG, Costa ES. Sexualidade da adolescente: anticoncepção e dst/aids. RBM. 2000;57:8-15.

9. Ministério da Saúde (BR). Gravidez na adolescência. [acesso em 18 mar 2006]. Disponível: http://migre. $\mathrm{me} / 51 \mathrm{LZC}$

10. De Barbieri MT. Certezas y malos entendidos sobre la categoría de gênero. In: Guzmán L, Pacheco G, compiladores. Estudios básicos de derechos humanos IV. San Jose: IDH, 1996. [acesso em 10 set 2010]. Disponível: http://www.bibliojuridica.org/libros/libro. htm?1=1838

11. Bozon $\mathrm{M}$, Heilborn ML. Iniciação à sexualidade: modos de socialização, interações de gênero e trajetórias individuais. In: Heilborn ML, Aquino EML, Bozon M, Knauth DR, organizadores. O aprendizado da sexualidade: reprodução e trajetórias sociais de jovens brasileiras. Rio de Janeiro: Garamond; 2006. p. 155-206.

12. Rena LCCB. Sexualidade e adolescência: as oficinas como prática pedagógica. Belo Horizonte: Autêntica; 2001.

13. Justo JS. O ficar na adolescência e paradigmas de relacionamento amoroso da contemporaneidade. Rev Dep Psicol. 2005;17(1):61-77.

14. Almeida MCC, Aquino EML, Gaffikin L, Magnani RJ. Uso de contracepção por adolescentes de escolas públicas na Bahia. Rev Saúde Pública. 2003;37(5):56675 .

15. Ferraz EA, Souza CT, Silva CFR, Costa, N. Iniciação sexual de jovens: análise de variáveis a partir de gênero. In: $15^{\circ}$ Encontro Nacional de Estudos Populacionais; Set. p.1-20; Caxambu, Brasil. 2006. [acesso em 20 set 2006]. Disponível: http://www.abep.nepo.unicamp.br/ encontro2006/docspdf/ABEP2006_561.pdf

16. Boruchovitch E. Fatores associados a não-utilização de anticoncepcionais na adolescência. Rev Saúde Pública. 1992;26(6):437-43.

17. Taquette SR, Vilhena MM, Paula MC. Doenças sexualmente transmissíveis na adolescência: estudo de fatores de risco. Rev Soc Bras Med Trop. 2004;37(3):2104.

18. Federação Brasileira das Sociedades de Ginecologia e Obstetrícia (FEBRASGO). Manual de anticoncepção on-line. 2001. [acesso em 14 mar 2006]. Disponível: http://www.anticoncepcao.org.br/html/manual/manual. htm

19. Lima H. Educação sexual para adolescentes: desvendando o corpo e os mitos. $3^{\mathrm{a}}$ ed. São Paulo: Iglu; 1994.

20. Louro GL. Gênero, sexualidade e educação: uma perspectiva pós-estruturalista. $2^{\mathrm{a}}$ ed. Petrópolis: Vozes; 1998. 